УДК 541.138.2

DOI: $10.17308 / \mathrm{kcmf} .2019 .21 / 1153$

Поступила в редакцию 26.06.2019

Подписана в печать 15.08.2019

\title{
КИНЕТИКА КАТОДНОГО ВЫДЕЛЕНИЯ ВОДОРОДА НА МОНОСИЛИЦИДЕ МАРГАНЦА В СЕРНОКИСЛОМ ЭЛЕКТРОЛИТЕ
}

\author{
(c) 2019 В. В. Пантелеева, И. С. Вотинов, И. С. Полковников, А. Б. Шеин ${ }^{\bowtie}$ \\ Пермский государственный национальный исследовательский университет \\ ул. Букирева, 15, 614990 Пермь, Российская Федерация
}

\begin{abstract}
Аннотация. Методами поляризационных и импедансных измерений изучена кинетика реакции выделения водорода на $\mathrm{MnSi}$-электроде в сернокислых растворах с различной концентрацией ионов водорода. Сделано предположение о механизме выделения водорода на силициде. Отмечено влияние тонкой оксидной пленки на кинетику выделения водорода на MnSi при невысоких катодных поляризациях.
\end{abstract}

Ключевые слова: силицид марганца $\mathrm{MnSi}$, реакция выделения водорода, сернокислый электролит, импеданс.

\section{ВВЕДЕНИЕ}

Реакция электрохимического образования водорода относится к электрокаталитическим процессам, скорость которых в значительной степени зависит от природы и структуры материала электрода [1, 2]. В качестве катализаторов реакции выделения водорода (р.в.в.) исследовались металлы, сплавы, интерметаллические и металлоподобные соединения, композиционные материалы [3-12] и др. Изучению кинетических закономерностей р.в.в. на силицидах переходных металлов посвящен ряд работ [3-6, 9, 12]; для некоторых силицидов отмечены более низкие перенапряжения выделения водорода по сравнению с соответствующими чистыми металлами."

Предметом настоящей работы является установление кинетики и механизма реакции выделения водорода на моносилициде марганца ( $\mathrm{MnSi}$ ) в сернокислом электролите, определение электрохимической активности $\mathrm{MnSi}$ в р.в.в.

\section{МАТЕРИАЛЫ И МЕТОДИКА ЭКСПЕРИМЕНТА}

Материалом для исследования служил моносилицид марганца $\mathrm{MnSi}$, который был получен методом Чохральского в печи для промышленного выращивания монокристаллов ОКБ-8093 («Редмет-8»).

Электрохимические измерения проведены при температуре $25{ }^{\circ} \mathrm{C}$ в условиях естествен-

$\triangle$ Шеин Анатолий Борисович, E-mail: ashein@psu.ru ной аэрации в неперемешиваемых растворах $0.5 \mathrm{M} \mathrm{H}_{2} \mathrm{SO}_{4} ; 0.35 \mathrm{M} \mathrm{H}_{2} \mathrm{SO}_{4}+0.15 \mathrm{M} \mathrm{Na}_{2} \mathrm{SO}_{4} ; 0.20 \mathrm{M}$ $\mathrm{H}_{2} \mathrm{SO}_{4}+0.30 \mathrm{M} \mathrm{Na}_{2} \mathrm{SO}_{4} ; 0.05 \mathrm{M} \mathrm{H}_{2} \mathrm{SO}_{4}+0.45 \mathrm{M}$ $\mathrm{Na}_{2} \mathrm{SO}_{4}$. Для приготовления растворов использовали деионизованную воду (удельное сопротивление воды - $18.2 \mathrm{MOhm} \cdot \mathrm{cm}$, содержание органического углерода $-4 \mathrm{mcg} / \mathrm{l})$, полученную с помощью системы очистки воды Milli-Q фирмы Millipore (Франция), и реактивы $\mathrm{H}_{2} \mathrm{SO}_{4}, \mathrm{Na}_{2} \mathrm{SO}_{4}$ марки «Х.ч.». Измерения проведены с помощью потенциостата-гальваностата с встроенным частотным анализатором Solartron 1280C фирмы Solartron Analytical (Великобритания) в электрохимической ячейке ЯСЭ-2 с разделенными пористой стеклянной диафрагмой катодным и анодным отделениями. В качестве электрода сравнения использовали насыщенный хлоридсеребряный электрод, в качестве вспомогательного электрода - платиновый электрод. Потенциалы в работе приведены относительно стандартного водородного электрода, плотности тока $i$ даны в расчете на единицу геометрической поверхности электрода.

Перед проведением измерений рабочую поверхность электрода шлифовали абразивными бумагами с последовательным уменьшением размера зерна, обезжиривали этиловым спиртом, ополаскивали рабочим раствором. После погружения в раствор электрод подвергали катодной поляризации при плотности тока $0.5 \mathrm{~mA} / \mathrm{cm}^{2}$ в течение $10 \mathrm{~min}$, затем выдерживали при потенциале разомкнутой цепи до ус- 
тановления стационарного значения, далее регистрировали спектры импеданса. Перед измерением спектров импеданса при каждом потенциале проводили потенциостатическую поляризацию электрода до установления практически постоянного значения тока, после чего начинали измерения импеданса при данном $E$ и более низких потенциалах, изменяя потенциал с определенным шагом. На основе полученных значений $i$ для данного значения $E$ строили катодные потенциостатические кривые. Диапазон используемых в импедансных измерениях частот $f(\omega / 2 \pi)$ - от $20 \mathrm{kHz}$ до $0.02 \mathrm{~Hz}$, амплитуда переменного сигнала (5-10) mV. В качестве критерия оценки схем на их пригодность для моделирования экспериментальных спектров импеданса использовали параметр $\chi^{2}$; эквивалентная схема считалась удовлетворительной при $\chi^{2}<10^{-3}$ (при использовании весовых коэффициентов, рассчитанных по экспериментальным значениям модуля импеданса).

\section{РЕЗУЛЬТАТЫ И ИХ ОБСУЖДЕНИЕ}

Катодные потенциостатические кривые $\mathrm{MnSi}$ электрода в растворах серной кислоты имеют однотипный вид и характеризуются наличием двух тафелевских участков с наклонами 0.17$0.18 \mathrm{~V}$ при $\lg i \approx-5.3 \ldots-4.4$ и $0.13-0.14 \mathrm{~V}$ при $\lg i \approx-4.4 \ldots-3.8$; при более высоких значениях $\lg i$ отмечается повышение наклона до $\sim 0.20-0.21 \mathrm{~V}$ (рис. 1). Постоянная $a$ в уравнении Тафеля для $\mathrm{MnSi}$ на первом участке равна $1.36-1.47 \mathrm{~V}$, на втором участке - $1.12-1.30 \mathrm{~V}$, то есть по классификации $[1,2]$ моносилицид марганца в сернокислом электролите относится к материалам с высоким перенапряжением выделения водорода.

Кинетические параметры реакции выделения водорода на $\mathrm{MnSi}$-электроде при $\lg i \approx-5.3 \ldots$ -3.8 близки к теоретическим для замедленной стадии переноса заряда (рис. 1, табл. 1) [1, 2].

Для уточнения механизма и кинетических закономерностей р.в.в. на MnSi-электроде в сернокислом электролите был использован метод спектроскопии электрохимического импеданса.
Дифференциальная емкость MnSi-электрода, измеренная при частоте переменного тока $f=10 \mathrm{kHz}$, в исследованной области составляет 4$7 \mu \mathrm{F} / \mathrm{cm}^{2}$, слабо зависит от потенциала и уменьшается при снижении кислотности среды (рис. 2). Дифференциальную емкость определяли из значений мнимой составляющей импеданса $Z^{\prime \prime}$ :

$$
C=-\frac{1}{\omega Z^{\prime \prime}},
$$

где $\omega$ - круговая частота переменного тока $(\omega=2 \pi f)$.

Пониженные значения дифференциальной емкости $\mathrm{MnSi}$-электрода по сравнению со значениями емкости, характерными для твердых металлических электродов $\left(20-30 \mu \mathrm{F} / \mathrm{cm}^{2}\right)$, могут быть связаны с низкой концентрацией носителей заряда в силициде. Однако в соответствии с

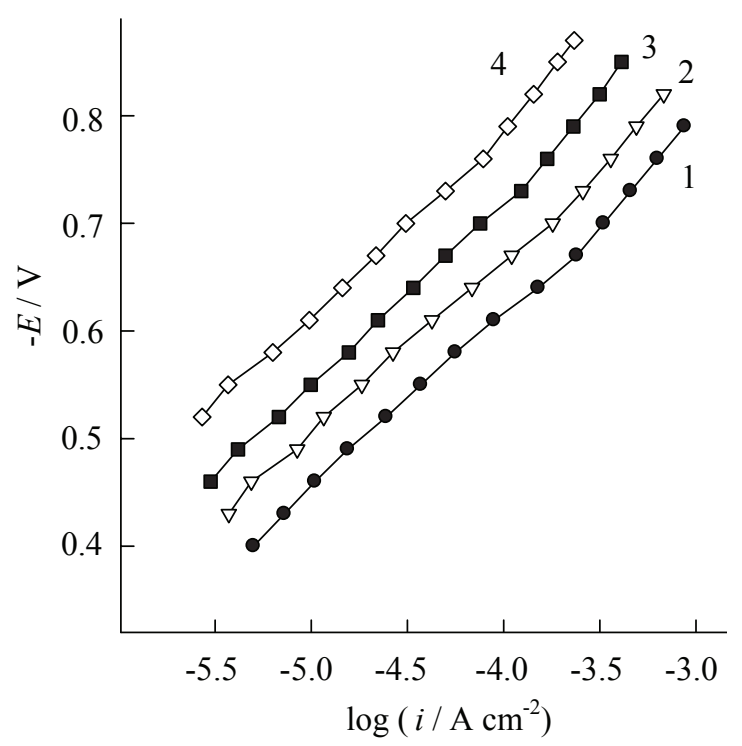

Рис. 1. Катодные потенциостатические кривые $\mathrm{MnSi}$-электрода в растворах: $1-0.5 \mathrm{M} \mathrm{H}_{2} \mathrm{SO}_{4}$; 2 $0.35 \mathrm{M} \mathrm{H}_{2} \mathrm{SO}_{4}+0.15 \mathrm{M} \mathrm{Na}_{2} \mathrm{SO}_{4} ; 3-0.20 \mathrm{M} \mathrm{H}_{2} \mathrm{SO}_{4}+$ $0.30 \mathrm{M} \mathrm{Na}_{2} \mathrm{SO}_{4} ; 4-0.05 \mathrm{M} \mathrm{H}_{2} \mathrm{SO}_{4}+0.45 \mathrm{M} \mathrm{Na}_{2} \mathrm{SO}_{4}$

[Fig. 1. Cathodic polarization curves for a $\mathrm{MnSi}$ electrode in solutions:

$1-0.5 \mathrm{M} \mathrm{H}_{2} \mathrm{SO}_{4} ; 2-0.35 \mathrm{M} \mathrm{H}_{2} \mathrm{SO}_{4}+0.15 \mathrm{M} \mathrm{Na}_{2} \mathrm{SO}_{4}$; $3-0.20 \mathrm{M} \mathrm{H}_{2} \mathrm{SO}_{4}+0.30 \mathrm{M} \mathrm{Na}_{2} \mathrm{SO}_{4} ; 4-0.05 \mathrm{M} \mathrm{H}_{2} \mathrm{SO}_{4}$ $\left.+0.45 \mathrm{M} \mathrm{Na}_{2} \mathrm{SO}_{4}\right]$

Таблица 1. Кинетические параметры реакции выделения водорода на $\mathrm{MnSi}$-электроде в растворах серной кислоты

[Table.1. Kinetic parameters of hydrogen evolution reaction for a MnSi electrode in sulfuric acid solutions]

\begin{tabular}{|c|c|c|c|c|}
\hline $\lg i\left[i, \mathrm{~A} / \mathrm{cm}^{2}\right]$ & $\left(\frac{\partial \lg i}{\partial \lg c_{\mathrm{H}^{+}}}\right)_{\eta}$ & $\left(\frac{\partial \lg i}{\partial \lg c_{\mathrm{H}^{+}}}\right)_{E}$ & $-\left(\frac{\partial \eta}{\partial \lg c_{\mathrm{H}^{+}}}\right)_{i}, \mathrm{~V}$ & $-\left(\frac{\partial E}{\partial \lg c_{\mathrm{H}^{+}}}\right)_{i}, \mathrm{~V}$ \\
\hline$-5.3 \ldots-4.4$ & 0.49 & 0.81 & 0.07 & 0.13 \\
\hline$-4.4 \ldots-3.8$ & 0.55 & 0.96 & 0.08 & 0.14 \\
\hline
\end{tabular}


[13] MnSi при комнатной температуре относится к металлическим проводникам и характеризуется удельным сопротивлением, не превышающим значений $35 \mathrm{\mu Ohm} \cdot \mathrm{m}$ [13-15]. По литературным данным, значения коэффициента Холла и его слабая зависимость от температуры для $\mathrm{MnSi}[16,17]$ также указывают на высокую проводимость силицида.

Низкие значения емкости силицида марганца могут быть обусловлены присутствием на его поверхности тонкой диэлектрической пленки диоксида кремния $\left(\mathrm{Si}+2 \mathrm{H}_{2} \mathrm{O} \rightarrow \mathrm{SiO}_{2}+4 \mathrm{H}^{+}+4 \mathrm{e}^{-}\right.$, $E^{0}=-0.86 \mathrm{~V}$ [18]). Согласно [3] $\mathrm{SiO}_{2}$ является устойчивым в кислых средах при невысоких катодных поляризациях.

Заключение о присутствии тонкого оксидного слоя на поверхности силицида объясняет снижение дифференциальной емкости с повышением рН электролита (рис. 2). Последнее может быть обусловлено меньшей скоростью химического растворения $\mathrm{SiO}_{2}$ в растворах с меньшей кислотностью. Согласно [19] скорость химического растворения различных форм диоксида кремния (кварц, аморфный оксид) минимальна при $\mathrm{pH} \sim(2-3)$ и возрастает при отклонении в обе стороны от этого значения.

Слабое изменение емкости с потенциалом (при $c_{\mathrm{H}^{+}}=$const) позволяет рассматривать

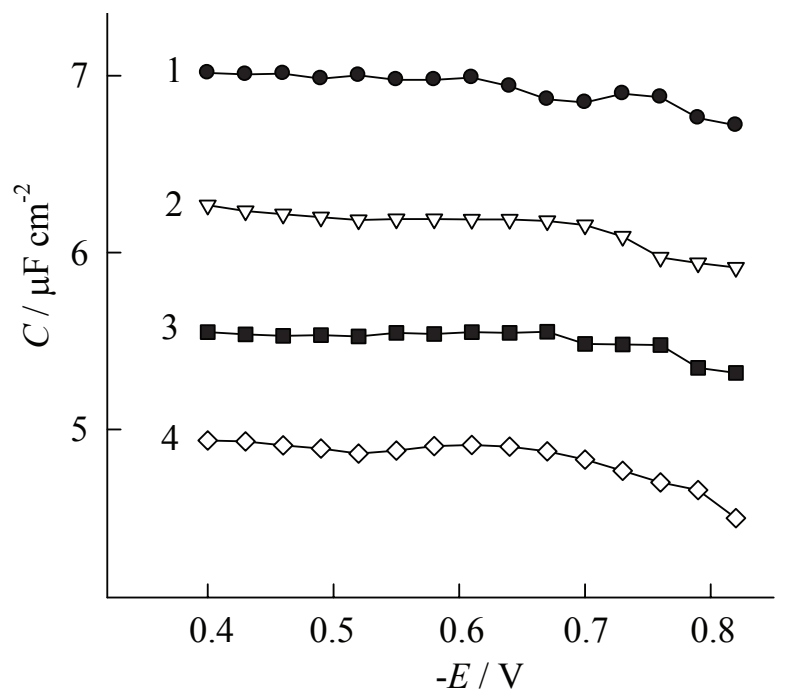

Рис. 2. Зависимость дифференциальной емкости $\mathrm{MnSi}$-электрода от потенциала при частоте 10 кГц в растворах: $1-0.5 \mathrm{M} \mathrm{H}_{2} \mathrm{SO}_{4} ; 2-0.35 \mathrm{M} \mathrm{H}_{2} \mathrm{SO}_{4}$ $+0.15 \mathrm{M} \mathrm{Na}_{2} \mathrm{SO}_{4} ; 3-0.20 \mathrm{M} \mathrm{H}_{2} \mathrm{SO}_{4}+0.30 \mathrm{M} \mathrm{Na}_{2} \mathrm{SO}_{4}$; $4-0.05 \mathrm{M} \mathrm{H}_{2} \mathrm{SO}_{4}+0.45 \mathrm{M} \mathrm{Na}_{2} \mathrm{SO}_{4}$

[Fig. 2. The dependence of differential capacitance of MnSi electrode on the potential at frequency of $10 \mathrm{kHz}$ in solutions: $1-0.5 \mathrm{M} \mathrm{H}_{2} \mathrm{SO}_{4} ; 2-0.35 \mathrm{M}$ $\mathrm{H}_{2} \mathrm{SO}_{4}+0.15 \mathrm{M} \mathrm{Na}_{2} \mathrm{SO}_{4} ; 3-0.20 \mathrm{M} \mathrm{H}_{2} \mathrm{SO}_{4}+0.30 \mathrm{M}$ $\left.\mathrm{Na}_{2} \mathrm{SO}_{4} ; 4-0.05 \mathrm{M} \mathrm{H}_{2} \mathrm{SO}_{4}+0.45 \mathrm{M} \mathrm{Na}_{2} \mathrm{SO}_{4}\right]$ $\lg i, E$-кривые (рис. 1) в предположении о постоянстве толщины оксидной пленки на электроде. В то же время снижение емкости (повышение толщины оксидного слоя) с уменьшением кислотности электролита может оказывать влияние на кинетику р.в.в. и искажать рассчитанные величины кинетических параметров (табл. 1). Авторами [20], исследовавшими влияние тонких пленок $\mathrm{SiO}_{2}(1.5-10 \mathrm{~nm})$, нанесенных на Pt-электрод, на кинетику р.в.в. в растворе серной кислоты, показано, что с увеличением толщины оксидной пленки плотность катодного тока (при $E=$ const) уменьшается.

Спектры импеданса $\mathrm{MnSi}$-электрода во всех исследованных растворах при $\lg i \approx-5.3 \ldots-3.8$ представляют собой емкостные полуокружности со смещенным центром; при $\lg i>-3.8$ в области низких частот на спектрах импеданса регистрируются отклонения от полуокружности, эти отклонения имеют вид почти прямолинейных участков и усиливаются с ростом рН электролита (рис. 3).

Для моделирования р.в.в. на $\mathrm{MnSi}$-электроде при $\lg i \approx-5.3 . . .-3.8$ в исследованных растворах использована эквивалентная электрическая схема, представленная на рис. $4 a$. В этой схеме: $R_{s}$ - сопротивление электролита, $R_{1}$ - сопротивление переноса заряда, сопротивление $R_{2}$ и емкость $C_{2}$ описывают адсорбцию атомарного водорода на поверхности электрода, $C_{1}$ - емкость двойного электрического слоя. Сопротивления $R_{1}$ и $R_{2}$ зависят от кинетических параметров обеих стадий р.в.в. [21]. В схеме на рис. $4 b$ вместо двойнослойной емкости используется элемент постоянной фазы СРЕ вает процесс заряжения двойного электрического слоя на неоднородной поверхности твердого электрода [22].

Импеданс элемента постоянной фазы равен:

$$
Z_{C P E}=Q^{-1}(j \omega)^{-p} \text {. }
$$

В этом соотношении при $p=1-\gamma$ элемент постоянной фазы представляет неидеальную емкость; $\gamma$ - величина, значительно меньше 1 (типично $\gamma<0.2$ ) [23].

Отсутствие в схемах на рис. 4 параллельной $R_{f} C_{f}$-цепочки $\left(R_{f}\right.$ и $C_{f}$ - сопротивление и емкость оксидной пленки), подключенной последовательно к межфазному импедансу и отражающей присутствие на поверхности силицида оксидной пленки, связано с небольшой толщиной пленки при катодных потенциалах; в результате вклад импеданса пленки в общий импеданс системы мал, и область релаксации в $R_{f} C_{f}$-цепочке с малыми $R_{f}$ и $C_{f}$ приходится на частоты выше $20 \mathrm{kHz}$. 

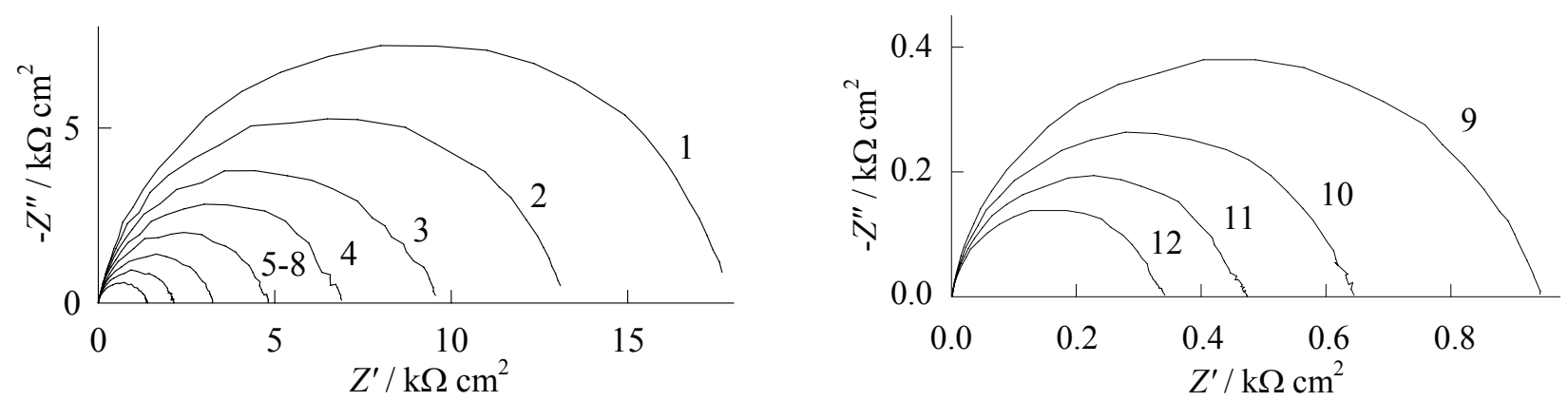

Рис. 3. Спектры импеданса MnSi-электрода в $0.20 \mathrm{M} \mathrm{H}_{2} \mathrm{SO}_{4}+0.30 \mathrm{M} \mathrm{Na}_{2} \mathrm{SO}_{4}$ при $E$, V: $1--0.49 ; 2--0.52$; $3--0.55 ; 4--0.58 ; 5--0.61 ; 6--0.64 ; 7--0.67 ; 8--0.70 ; 9--0.73 ; 10--0.76 ; 11--0.79 ; 12--0.82$ [Fig. 3. Impedance spectra of MnSi electrode in $0.20 \mathrm{M} \mathrm{H}_{2} \mathrm{SO}_{4}+0.30 \mathrm{M} \mathrm{Na}_{2} \mathrm{SO}_{4}$ at $E, \mathrm{~V}: 1--0.49 ; 2--0.52$; $3--0.55 ; 4--0.58 ; 5--0.61 ; 6--0.64 ; 7--0.67 ; 8--0.70 ; 9--0.73 ; 10--0.76 ; 11--0.79 ; 12--0.82]$
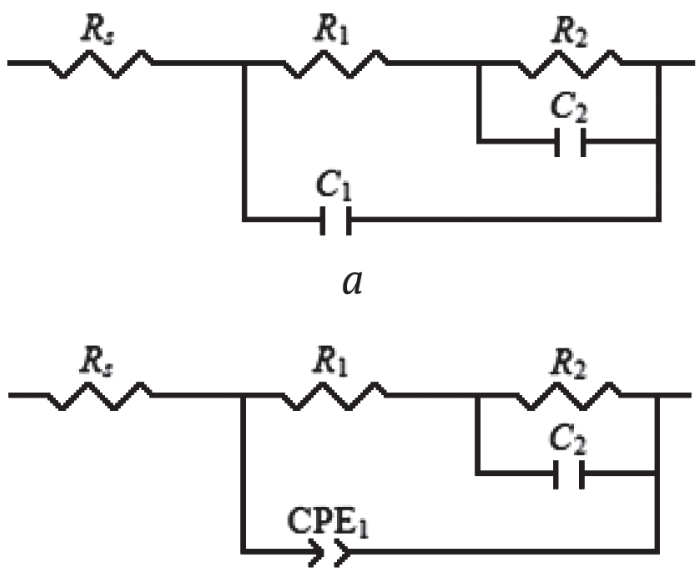

$b$

Рис. 4. Эквивалентные электрические схемы для MnSi-электрода в сернокислом растворе в области потенциалов выделения водорода

[Fig. 4. Equivalent electrical circuits for a MnSi electrode in a sulfuric acid solution in the range of hydrogen evolution potentials]

Применение нелинейного метода наименьших квадратов (программа ZView2) показало, что эквивалентная схема на рис. $4 b$ удовлетворительно описывает экспериментальные спектры импеданса $\mathrm{MnSi}$-электрода, полученные при $\lg i<-3.8$. Критерий $\chi^{2}$, вычисленный в ZView2, для схемы на рис. $4 b$ составляет $(0.9-2.4) \cdot 10^{-4}$; ошибка определения значений параметров схемы не превышает 5 \%. Значения параметров эквивалентной схемы для раствора $0.20 \mathrm{M} \mathrm{H}_{2} \mathrm{SO}_{4}+$ $0.30 \mathrm{M} \mathrm{Na}_{2} \mathrm{SO}_{4}$ приведены в табл. 2 .

Результаты определения численных значений параметров $R_{1}, R_{2}, C_{2}$ эквивалентной схемы на рис. $4 b$ и произведений $i R_{1}, i R_{2}$ проанализированы в зависимости от потенциала в полулогарифмических координатах (рис. 5, табл. 3). В области lgi $\approx-5.3 \ldots-3.8$ экспериментальные зна-

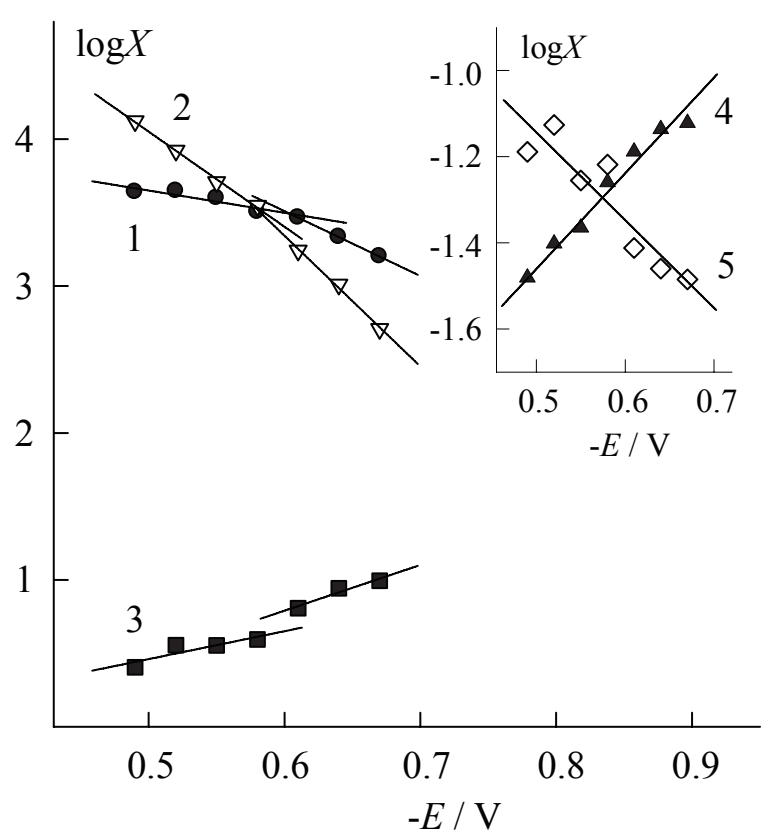

Рис. 5. Зависимости $\lg X\left(X: 1-R_{1}, 2-R_{2}, 3-C_{2}\right.$, $4-i R_{1}, 5-i R_{2}$ ) от потенциала $\mathrm{MnSi}$-электрода в растворе $0.20 \mathrm{M} \mathrm{H}_{2} \mathrm{SO}_{4}+0.30 \mathrm{M} \mathrm{Na}_{2} \mathrm{SO}_{4}$. Единицы измерения: $R_{1}$ и $R_{2}$ - в $\mathrm{Ohm} \cdot \mathrm{cm}^{2}, C_{2}-$ в $\mu \mathrm{F} / \mathrm{cm}^{2}$, произведения $i R_{1}$ и $i R_{2}-$ в V

[Fig. 5. Dependences of $\log X\left(X: 1-R_{1}, 2-R_{2}, 3-C_{2}\right.$, $4-i R_{1}, 5-i R_{2}$ ) on $E$ for a $\mathrm{MnSi}$ electrode in $0.20 \mathrm{M}$ $\mathrm{H}_{2} \mathrm{SO}_{4}+0.30 \mathrm{M} \mathrm{Na}_{2} \mathrm{SO}_{4}$. Resistances $R_{1}$ and $R_{2}$ are in $\Omega \mathrm{cm}^{2}$, capacitance $C_{2}$ in $\mu \mathrm{F} \mathrm{cm}^{-2}, i R_{1}$ and $i R_{2}-$ in V]

чения наклонов $(\partial \lg X / \partial E)_{\mathcal{c}_{\mathrm{H}^{+}}}$, где $X=R_{1}, R_{2}, C_{2}$, $i R_{1}, i R_{2}$, близки к теоретическим значениям наклонов для механизма разряд - электрохимическая десорбция, в котором обе стадии необратимы и коэффициенты переноса стадий не равны $[24,25]$. Линейность $\lg \left(R_{1}, R_{2}, C_{2}\right), E$-зависимостей свидетельствует о выполнении изотермы адсорбции Ленгмюра для адсорбированного атомарного водорода [24]. 
Таблица 2. Значения параметров эквивалентной схемы на рис. $4 b$ для $\mathrm{MnSi}$-электрода в растворе $0.20 \mathrm{M} \mathrm{H}_{2} \mathrm{SO}_{4}+0.30 \mathrm{M} \mathrm{Na}_{2} \mathrm{SO}_{4}$

[Table 2. The values of the equivalent circuit parameters in Fig. $4 b$ for a MnSi electrode in solution $\left.0.20 \mathrm{M} \mathrm{H}_{2} \mathrm{SO}_{4}+0.30 \mathrm{M} \mathrm{Na}_{2} \mathrm{SO}_{4}\right]$

\begin{tabular}{|c|c|c|c|c|c|}
\hline$-E, \mathrm{~V}$ & $\begin{array}{c}R_{1}, \\
\mathrm{Ohm} \cdot \mathrm{cm}^{2}\end{array}$ & $\begin{array}{c}R_{2}, \\
\mathrm{Ohm} \cdot \mathrm{cm}^{2}\end{array}$ & $\begin{array}{c}C_{2} \cdot 10^{6}, \\
\mathrm{~F} \cdot \mathrm{cm}^{-2}\end{array}$ & $\begin{array}{c}Q_{1} \cdot 10^{5}, \\
\mathrm{~F} \cdot \mathrm{cm}^{-2} \cdot \mathrm{s}^{\left(p_{1}-1\right)}\end{array}$ & $p_{1}$ \\
\hline 0.49 & 4364 & 13230 & 2.54 & 2.08 & 0.895 \\
\hline 0.52 & 4434 & 8359 & 3.60 & 2.02 & 0.898 \\
\hline 0.55 & 3959 & 5095 & 3.58 & 2.00 & 0.900 \\
\hline 0.58 & 3197 & 3514 & 3.94 & 1.95 & 0.902 \\
\hline 0.61 & 2919 & 1744 & 6.42 & 1.89 & 0.905 \\
\hline 0.64 & 2155 & 1023 & 8.78 & 1.87 & 0.906 \\
\hline 0.67 & 1587 & 510.4 & 9.87 & 1.83 & 0.909 \\
\hline
\end{tabular}

Таблица 3. Величины наклонов $(\partial \lg X / \partial E)_{c_{\mathrm{H}^{+}}}\left(X=R_{1}, R_{2}, C_{2}, i R_{1}, i R_{2}\right)$ для $\mathrm{MnSi-электрода} \mathrm{в} \mathrm{растворах} \mathrm{серной} \mathrm{кислоты}$

[Table 3. Slope values $(\partial \lg X / \partial E)_{\mathcal{C}_{\mathrm{H}^{+}}}\left(X=R_{1}, R_{2}, C_{2}, i R_{1}, i R_{2}\right)$ for a $\mathrm{MnSi}$ electrode in sulfuric acid solutions]

\begin{tabular}{|c|c|c|c|c|c|c|}
\hline \multirow{2}{*}{ Раствор } & $\lg i\left[i, \mathrm{~A} / \mathrm{cm}^{2}\right]$ & $\left(\frac{\partial \lg R_{1}}{\partial E}\right)_{c_{\mathrm{H}^{+}}}, \mathrm{V}^{-1}\left(\frac{\partial \lg R_{2}}{\partial E}\right)_{\mathrm{H}^{+}}, \mathrm{V}^{-1}$ & $\left(\frac{\partial \lg C_{2}}{\partial E}\right)_{\mathrm{H}^{+}}, \mathrm{V}^{-1}\left(\frac{\partial \lg i R_{1}}{\partial E}\right)_{\mathrm{H}^{+}}, \mathrm{V}^{-1}\left(\frac{\partial \lg i R_{2}}{\partial E}\right)_{c_{\mathrm{H}^{+}}}, \mathrm{V}^{-1}$ \\
\hline $0.5 \mathrm{M} \mathrm{H}_{2} \mathrm{SO}_{4}$ & $-5.3 \ldots-4.4$ & 3.5 & 5.8 & -4.1 & -1.2 & 1.6 \\
\cline { 2 - 7 } & $-4.4 \ldots-3.8$ & 7.4 & 8.2 & -6.4 & -0.2 & 1.4 \\
\hline $0.35 \mathrm{M} \mathrm{H}_{2} \mathrm{SO}_{4}+$ & $-5.3 \ldots-4.4$ & 3.4 & 5.7 & -2.5 & -1.2 & 0.7 \\
\cline { 2 - 7 } $0.15 \mathrm{M} \mathrm{Na}_{2} \mathrm{SO}_{4}$ & $-4.4 \ldots-3.8$ & 4.8 & 9.5 & -4.9 & -0.9 & 1.3 \\
\hline $0.20 \mathrm{M} \mathrm{H}_{2} \mathrm{SO}_{4}+$ & $-5.3 \ldots-4.4$ & 3.2 & 6.5 & -2.3 & -1.4 & 0.7 \\
\hline $0.30 \mathrm{M} \mathrm{Na}_{2} \mathrm{SO}_{4}$ & $-4.4 \ldots-3.8$ & 4.4 & 9.0 & -4.4 & -1.1 & 1.2 \\
\hline $0.05 \mathrm{M} \mathrm{H}_{2} \mathrm{SO}_{4}+$ & $-5.3 \ldots-4.4$ & 3.1 & 5.5 & -2.6 & -1.4 & 1.3 \\
\hline $0.45 \mathrm{M} \mathrm{Na}_{2} \mathrm{SO}_{4}$ & $-4.4 \ldots-3.8$ & 4.2 & 7.8 & -4.3 & -2.1 & 1.9 \\
\hline
\end{tabular}

Пониженные значения производных $(\partial \lg X / \partial E)_{c_{\mathrm{H}^{+}}}$согласно [26] могут быть связаны с тем, что одновременно с реакцией выделения водорода протекает реакция абсорбции водорода материалом электрода. Отсутствие дополнительной временной константы, характеризующей абсорбцию водорода, на спектрах импеданса MnSi-электрода (рис. 3) при $\lg i \approx-5.3 \ldots-3.8$ свидетельствует о том, что скорость-определяющим процессом при абсорбции водорода, по-видимому, является переход водорода из адсорбированного состояния в абсорбированное. В этом случае сопротивление $R_{2}$ в эквивалентных схемах на рис. 4 выражается через адсорбционное сопротивление $R_{a d s}$ и сопротивление абсорбции $R_{a b s}: R_{2}=\frac{R_{a d s} R_{a b s}}{R_{a d s}+R_{a b s}}$. При $\lg i>-3.8$ наводороживание материала электрода, по-видимому, протекает более интенсивно, о чем говорит появление на спектрах импеданса в области низких частот отклонений от полуокружности (рис. 3).

\section{выводы}

1. Реакция выделения водорода на силициде марганца в сернокислом электролите протекает по маршруту разряд - электрохимическая десорбция, обе стадии необратимы, коэффициенты переноса стадий не равны; одновременно с реакцией выделения водорода протекает реакция абсорбции водорода с кинетическим контролем. Для адсорбированного атомарного водорода выполняется изотерма адсорбции Ленгмюра.

2. Отмечается влияние тонкой оксидной пленки на кинетику выделения водорода на MnSi при невысоких катодных поляризациях.

\section{КОНФЛИКТ ИНТЕРЕСОВ}

Авторы декларируют отсутствие явных и потенциальных конфликтов интересов, связанных с публикацией настоящей статьи. 


\section{СПИСОК ЛИТЕРАТУРЫ}

1. Ротинян А. Л., Тихонов К. И., Шошина И. А. Теоретическая электрохимия. Ленинград, Химия, 1981,424 c.

2. Антропов Л. И. Теоретическая электрохимия. М.: Высшая школа, 1984, 519 с.

3. Shamsul Huq A. K. M., Rosenberg A. J. J. Electrochemical behavior of nickel compounds // Electrochem. Soc., 1964, v. 111(3), p. 270. DOI: 10.1149/1.2426107

4. Vijh A. K., Belanger G., Jacques R. Electrochemical reactions oh iron silicide surfaces in sulphuric acid // Materials Chemistry and Physics, 1988, v. 20(6), pp. 529-538. DOI: 10.1016/0254-0584(88)90086-7

5. Vijh A. K., Belanger G., Jacques R. Electrochemical activity of silicides of some transition metals for the hydrogen evolution reaction in acidic solutions // Int. J. Hydrogen Energy, 1990, v. 15(11), pp. 789-794. DOI: 10.1016/0360-3199(90)90014-P

6. Шеин А. Б. Электрохимия силицидов и германидов переходных металлов. Пермь, Перм. гос. ун-т, 2009. $269 \mathrm{c}$.

7. Vigdorovich V. I., Tsygankova L. E., Gladysheva I. E., Kichigin V. I. Kinetics of hydrogen evolution from acidic solutions on pressed micro graphite electrodes modified with carbon nanotubes. II. Impedance studies // Protection of Metals and Physical Chemistry of Surfaces, 2012, v. 48(4), pp. 438-443. DOI: 10.1134/ S2070205112040181

8. Meyer S., Nikiforov A. V., Petrushina I. M., Kohler K., Christensen E., Jensen J.O., Bjerrum N. J. Transition metal carbides (WC, $\mathrm{Mo}_{2} \mathrm{C}, \mathrm{TaC}, \mathrm{NbC}$ ) as potential electrocatalysts for the hydrogen evolution reaction (HER) at medium temperatures // Int. J. Hydrogen Energy, 2015, v. 40(7), pp. 2905-2911. DOI: 10.1016/j.ijhydene.2014.12.076

9. Кичигин В. И., Шеин А. Б., Шамсутдинов А. Ш. Кинетика катодного выделения водорода на моносилициде железа а кислых и щелочных средах // Конденсированные среды и межфазные границы, 2016, т. 18, № 3, с. 326-337. URL: https://journals.vsu. $\mathrm{ru} / \mathrm{kcmf} /$ article/view/140/98

10. Eftekhari A. Electrocatalysts for hydrogen evolution reaction // International Journal of Hydrogen Energy, 2017,v. 42(16), pp. 11053-11077.DOI: 10.1016/ j.ijhydene.2017.02.12511.

11. Schalenbach M., Speck F. D., Ledendecker M., Kasian O., Goehl D., Mingers A. M., Breitbach B., Springer H., Cherevko S., Mayrhofer K. J. J. Nickelmolybdenum alloy catalysts for the hydrogen evolution reaction: Activity and stability revised // Electrochimica Acta, 2018, v. 259, pp. 1154-1161. DOI: 10.1016/j.electacta.2017.11.069

12. Кузьминых М. М., Пантелеева В. В., Шеин А. Б. Катодное выделение водорода на дисилициде железа. II Кислая среда // Известия вузов.
Химия и хим. технология, 2019, т. 62(2), с. 59-64.DOI: 10.6060/ivkkt.20196202.5750

13. Самсонов Г. В., Дворина Л. А., Рудь Б. М. Силициды. М.: Металлургия, 1979, 272 с.

14. Самсонов Г. В., Виницкий И. М. Тугоплавкие соединения. М.: Металлургия, 1976, 560 с.

15. Yamasaki T., Okada S., Kamamoto K., Kudou K. Crystal Growth and properties of manganese-silicon system compounds by high-temperature tin solution method // Pacific Science Review, 2012, v. 14(3), pp. 275.

16. Lee M., Onose Y., Tokura Y., Ong N.P. Hidden constant in the anomalous Hall effect of high-purity magnet MnSi // Phys. Rev. B., 2007, v. 75(17), p. 172403. DOI: 10.1103/PhysRevB.75.172403

17. Neubauer A., Pfleiderer C., Binz B., Rosch A., Ritz R., Niklowitz P. G., Boni P. Topological Hall effect in the a phase of MnSi // Phys. Rev. Lett., 2009, v. 102(18), pp. 186602. DOI: 10.1103/PhysRevLett.102.186602

18. Сухотин А. М. Справочник по электрохимии. Ленинград, Химия, 1981, 488 с.

19. Zhang X. G. Electrochemistry of silicon and its oxide. Kluwer Academic/Plenum Publishers, New York, $2001.510 \mathrm{p}$.

20. Xu X., Bojkov H., Goodman D. W. Electrochemical study of ultrathin silica films supported on a platinum substrate // J. Vac. Sci. Technol., 1994, v. A12(4), pp. 1882-1885. DOI: 10.1116/1.579022

21. Harrington D. A., Conway B. E. ac Impedance of Faradaic reactions involving electrosorbed intermediates-I. Kinetic theory // Electrochim. Acta, v. 32(12), pp. 1703-1712. DOI: 10.1016/00134686(87)80005-1

22. Orazem M. E., Tribollet B. Electrochemical Impedance Spectroscopy. J. Wiley and Sons, Hoboken, New York, 2008, 533 p.

23. Кичигин В. И., Шерстобитова И. Н., Шеин А. Б. Импеданс электрохимических и коррозионных систем: учеб. пособие по специкурсу. Пермь, Перм. гос. ун-т, 2009, 239 с.

24. Kichigin V. I., Shein A. B. Diagnostic criteria for hydrogen evolution mechanisms in electrochemical impedance spectroscopy // Electrochemica Acta, 2014 , v. 138 , pp. 325-333. DOI: 10.1016/j.electacta.2014.06.114

25. Кичигин В. И., Шеин А. Б. Дополнительные критерии механизма реакции выделения водорода в методе импеданстной спектроскопии // Becmник Пермского университета. Сер. Химия, 2018, т. 8, вып. 3, с. 316-324. DOI: 10.17072/2223-1838-20183-316-324

26. Kichigin V. I., Shein A. B. Influence of hydrogen absorption on the potential dependence of the Faradaic impedance parameters of hydrogen evolution reaction // Electrochemica Acta, 2016, v. 201, pp. 233239. DOI: 10.1016/j.electacta.2016.03.194 
UDC 541.138.2

DOI: $10.17308 / \mathrm{kcmf} .2019 .21 / 1153$

Received 26.06.2019

Accepted 15.08.2019

\title{
KINETICS OF CATHODIC HYDROGEN EVOLUTION ON MANGANESE MONOSILICIDE IN SULFURIC ACID ELECTROLYTE
}

\author{
(c) 2019 V. V. Panteleeva , I. S. Votinov, I. S. Polkovnikov, A. B. Shein \\ Perm State University \\ 15 Bukirev str., 614990 Perm, Russian Federation
}

\begin{abstract}
Purpose. The purpose of the work is to investigate the kinetics of the hydrogen evolution reaction (h.e.r.) on the $\mathrm{MnSi}$ electrode in solutions $x \mathrm{M} \mathrm{H}_{2} \mathrm{SO}_{4}+(0.5-x) \mathrm{M} \mathrm{Na}_{2} \mathrm{SO}_{4}(x=0.5 ; 0.35 ; 0.20$; 0.05 ).

Methods and methodology. Methods used in the work are polarization and impedance measurements. The research material is manganese silicide $\mathrm{MnSi}$, which was obtained by the Czochralski method. The range of frequencies $f(\omega / 2 \pi)$ used in impedance measurements is from $20 \mathrm{kHz}$ to $0.02 \mathrm{~Hz}$, the amplitude of the alternating signal is $(5-10) \mathrm{mV}$.

Results. The following results have been obtained. Cathodic polarization curves of the MnSi electrode have two Tafel plots with slopes of $0.17-0.18 \mathrm{~V}$ at $\lg i \approx-5.3 \ldots-4.4$ and $0.13-0.14 \mathrm{~V}$ at $\lg i \approx-4.4 \ldots-3.8$. The constant $a$ in the Tafel equation for MnSi is equal to $1.36-1.47 \mathrm{~V}$ for the first plot, and 1.12-1.30 V for the second plot. Silicide in sulfuric acid electrolyte refers to materials with high overpotential of hydrogen evolution. Kinetic parameters of h.e.r. $(\partial E / \partial \lg i)_{c_{\mathrm{H}^{+}}}$, $\left(\partial E / \partial \lg c_{\mathrm{H}^{+}}\right)_{i},\left(\partial \eta / \partial \lg c_{\mathrm{H}^{+}}\right)_{i},\left(\partial \lg i / \partial \lg c_{\mathrm{H}^{+}}\right)_{E},\left(\partial \lg i / \partial \lg c_{\mathrm{H}^{+}}\right)_{\eta}$ on MnSi are close to theoretical for the slow stage of charge transfer.

The impedance spectra of the MnSi electrode in the studied solutions at $\lg i \approx-5.3 \ldots-3.8$ are capacitive semi-circles with an offset center. The impedance spectra are satisfactory (the $\chi^{2}$ criterion is $\sim 10^{-4}$; the error in determining the values of the circuit parameters does not exceed $5 \%$ ) described by an equivalent electrical circuit whose Faraday impedance consists of in seriesconnected resistances of charge transfer $R_{1}$ and a parallel $R_{2} C_{2}$ chain corresponding to the adsorption of atomic hydrogen on the electrode surface; the impedance of the double-layer capacitance is modeled by the constant phase element $\mathrm{CPE}_{1}$.

The results of determining the values of the parameters $R_{1}, R_{2}, C_{2}$ of the equivalent circuit and also $i R_{1}, i R_{2}$ have been analyzed depending on the potential in semi-logarithmic coordinates.

Conclusions. Conclusions have been made that in the region lg $i \approx-5.3 \ldots-3.8$, the experimental values of the slopes $(\partial \lg X / \partial E)_{\mathrm{CH}^{+}}$, where $X=R_{1}, R_{2}, C_{2}, i R_{1}, i R_{2}$, are close to the theoretical values of the slopes for the discharge - electrochemical desorption mechanism, in which both stages are irreversible and the transfer coefficients of the stages are not equal. Simultaneously with the hydrogen evolution reaction, the hydrogen absorption reaction proceeds with kinetic control; for adsorbed atomic hydrogen the Langmuir adsorption isotherm is performed.
\end{abstract}

Keywords: manganese silicide MnSi, hydrogen evolution reaction, sulfuric acid electrolyte, impedance.

\section{CONFLICT OF INTEREST}

The authors declare the absence of obvious and potential conflicts of interest related to the publication of this article."

$\square$ Shein Anatoliy B., e-mail: ashein@psu.ru

\section{REFERENCES}

1. Rotinyan A. L., Tikhonov K. I., Shoshina I. A. Teoreticheskaya elektrokhimiya [Theoretical Electrochemistry]. Leningrad, Khimiya Publ., 1981, 424 p. (in Russ.)

2. Antropov L. I. Teoreticheskaya elektrokhimiya [Theoretical Electrochemistry]. Moscow, Vysshaya shkola Publ., 1984, 519 p. (in Russ.) 
3. Shamsul Huq A. K. M., Rosenberg A. J. J. Electrochemical behavior of nickel compounds. Electrochem. Soc., 1964, v. 111(3), p. 270. DOI: 10.1149/1.2426107

4. Vijh A. K., Belanger G., Jacques R. Electrochemical reactions oh iron silicide surfaces in sulphuric acid. Materials Chemistry and Physics, 1988, v. 20(6), pp. 529-538. DOI: 10.1016/0254-0584(88)90086-7

5. Vijh A. K., Belanger G., Jacques R. Electrochemical activity of silicides of some transition metals for the hydrogen evolution reaction in acidic solutions. Int. J. Hydrogen Energy, 1990, v. 15(11), pp. 789-794. DOI: 10.1016/0360-3199(90)90014-P

6. Shein A. B. Elektrokhimiya silitsidov i germanidov perekhodnykh metallov [Electrochemistry of silicides and germanides of transition metals]. Perm', Perm. gos. un-t Publ., 2009, 269 p. (in Russ.)

7. Vigdorovich V. I., Tsygankova L. E., Gladysheva I. E., Kichigin V. I. Kinetics of hydrogen evolution from acidic solutions on pressed micro graphite electrodes modified with carbon nanotubes. II. Impedance studies. Protection of Metals and Physical Chemistry of Surfaces, 2012, v. 48(4), pp. 438-443. DOI: 10.1134/ S2070205112040181

8. Meyer S., Nikiforov A. V., Petrushina I. M., Kohler K., Christensen E., Jensen J. O., Bjerrum N. J. Transition metal carbides (WC, $\mathrm{Mo}_{2} \mathrm{C}, \mathrm{TaC}, \mathrm{NbC}$ ) as potential electrocatalysts for the hydrogen evolution reaction (HER) at medium temperatures. Int. J. Hydrogen Energy, 2015, v. 40(7), pp. 2905-2911. DOI: 10.1016/j.ijhydene.2014.12.076

9. Kichigin V. I., Shein A. B., Shamsutdinov A. Sh. The kinetics of cathodic hydrogen evolution on iron monosilicide in acid and alkaline solutions. Kondensirovannye sredy i mezhfaznye granitsy [Condensed Matter and Interphases], 2016, v. 18(3), pp. 326-337. URL: https://journals.vsu.ru/kcmf/article/view/140/98 (in Russ.)

10. Eftekhari A. Electrocatalysts for hydrogen evolution reaction. International Journal of Hydrogen Energy, 2017,v. 42(16),pp. 11053-11077.DOI: 10.1016/ j.ijhydene.2017.02.125

11. Schalenbach M., Speck F. D., Ledendecker M., Kasian O., Goehl D., Mingers A. M., Breitbach B., Springer H., Cherevko S., Mayrhofer K. J. J. Nickelmolybdenum alloy catalysts for the hydrogen evolution reaction: Activity and stability revised. Electrochimica Acta, 2018, v. 259, pp. 1154-1161. DOI: 10.1016/j.electacta.2017.11.069

12. Kuz'minykh M. M., Panteleeva V. V., Shein A. B. Cathodic hydrogen evolution on iron disilicide. II. Acidic solution. Izvestiya vuzov. Khimiya i khim. tekhnologiya, 2019, v. 62(2), pp. 59-64. DOI: 10.6060/ivkkt.20196202.5750 (in Russ.)

13. Samsonov G. V., Dvorina L. A., Rud' B.M. Silitsidy [Silicides]. Moscow, Metallurgiya Publ., 1979, 272 p. (in Russ.)
14. Samsonov G. V., Vinitskii I. M. Tugoplavkie soedineniya [Refractory compounds]. Moscow, Metallurgiya Publ., 1976, 560 p. (in Russ.)

15. Yamasaki T., Okada S., Kamamoto K., Kudou K. Crystal Growth and properties of manganese-silicon system compounds by high-temperature tin solution method. Pacific Science Review, 2012, v. 14(3), pp. 275.

16. Lee M., Onose Y., Tokura Y., Ong N. P. Hidden constant in the anomalous Hall effect of high-purity magnet MnSi. Phys. Rev. B., 2007, v. 75(17), p. 172403. DOI: 10.1103/PhysRevB.75.172403

17. Neubauer A., Pfleiderer C., Binz B., Rosch A., Ritz R., Niklowitz P. G., Boni P. Topological Hall effect in the a phase of MnSi. Phys. Rev. Lett., 2009, v. 102(18), pp. 186602. DOI: 10.1103/PhysRevLett.102.186602

18. Sukhotin A. M. Spravochnik po elektrokhimii [Handbook of electrochemistry]. Leningrad, Khimiya Publ., 1981, 488 p. (in Russ.)

19. Zhang X. G. Electrochemistry of silicon and its oxide. Kluwer Academic/Plenum Publishers, New York, 2001. $510 \mathrm{p}$.

20. Xu X., Bojkov H., Goodman D. W. Electrochemical study of ultrathin silica films supported on a platinum substrate. J. Vac. Sci. Technol., 1994, v. A12(4), pp. 1882-1885. DOI: 10.1116/1.579022

21. Harrington D. A., Conway B. E. ac Impedance of Faradaic reactions involving electrosorbed intermediates - I. Kinetic theory. Electrochim. Acta, v. 32(12), pp. 1703-1712. DOI: 10.1016/00134686(87)80005-1

22. Orazem M. E., Tribollet B. Electrochemical Impedance Spectroscopy. J. Wiley and Sons, Hoboken, New York, 2008, 533 p.

23. Kichigin V. I., Sherstobitova I. N., Shein A. B. Impedans elektrokhimicheskikh $i$ korrozionnykh sistem: ucheb. posobie po spetskursu [The impedance of electrochemical and corrosion systems: textbook. special course allowance]. Perm', Perm. gos. un-t Publ., 2009, 239 p. (in Russ.)

24. Kichigin V. I., Shein A. B. Diagnostic criteria for hydrogen evolution mechanisms in electrochemical impedance spectroscopy. Electrochemica Acta, 2014, v. 138, pp. 325-333. DOI: 10.1016/j.electacta.2014.06.114

25. Kichigin V. I., Shein A. B. Additional criteria for the mechanism of hydrogen evolution reaction in the impedance spectroscopy method. Vestnik Permskogo Universiteta. Ser. Khimiya, 2018, v. 8, iss. 3, pp.316-324. DOI: 10.17072/2223-1838-2018-3-316-324 (in Russ.)

26. Kichigin V. I., Shein A. B. Influence of hydrogen absorption on the potential dependence of the Faradaic impedance parameters of hydrogen evolution reaction. Electrochemica Acta, 2016, v. 201, pp. 233239. DOI: 10.1016/j.electacta.2016.03.194 
Пантелеева Виктория Вячеславовна - к. х. н., доцент кафедры физической химии химического факультета, Пермский государственный национальный исследовательский университет, Пермь, Российская Федерация; e-mail:vikpant@ mail.ru. ORCID iD 0000-0002-1506-6665.

Вотинов Илья Сергеевич - магистрант кафедры физической химии химического факультета, Пермский государственный национальный исследовательский университет, Пермь, Российская Федерация; e-mail: Anatoly173@yandex.ru. ORCID iD 0000-0002-9027-8924.

Полковников Игорь Сергеевич - магистрант кафедры физической химии химического факультета, Пермский государственный национальный исследовательский университет, Пермь, Российская Федерация; e-mail: Anatoly173@yandex.ru. ORCID iD 0000-0003-4381-6467.

Шеин Анатолий Борисович - д. х. н., профессор, зав. кафедрой физической химии химического факультета, Пермский государственный национальный исследовательский университет, Пермь, Российская Федерация; e-mail: ashein@ psu.ru. ORCID iD 0000-0002-2102-0436.
Panteleeva Viktoria V. - Dr. Sci. (Chem), Associate Professor, Department of Physical Chemistry, Perm State University, Perm, Russian Federation; e-mail: vikpant@mail.ru. ORCID iD 0000-00021506-6665.

Votinov Ilya S. - Master student, Department of Physical Chemistry, Perm State University, Perm, Russian Federation; e-mail: Anatoly173@yandex. ru. ORCID iD 0000-0002-9027-8924.

Polkovnikov Igor S. - Master student, Department of Physical Chemistry, Perm State University, Perm, Russian Federation; e-mail: Anatoly173@yandex. ru. ORCID iD 0000-0003-4381-6467.

Shein Anatoliy B. - Dr. Sci. (Chem.), Professor, Head of the Department of Physical Chemistry, Perm State University, Perm, Russian Federation; e-mail: ashein@psu.ru. ORCID iD 0000-00022102-0436. 